\title{
La competencia pragmática en adolescentes. Un estudio de la atenuación en actos de rechazo
}

Pragmatic competence in adolescents. A mitigation study in refusal speech acts

ÀNGELA MAGRANER MifSUd

UNIVERSITAT DE VALÈNCIA

Artículo recibido el / Article received: 2018-11-09

Artículo aceptado el / Article accepted: 2019-01-19

RESUMEN: En el presente trabajo se ha llevado a cabo un estudio de la atenuación pragmática a partir del análisis de un corpus de muestras escritas que recogen una actividad de simulación o role-play propuesta a estudiantes adolescentes (15-18 años): la escritura de un WhatsApp en la que se rechaza una invitación. El objetivo de este estudio es, por una parte, observar el uso que hacen los adolescentes de la estrategia pragmática de la atenuación, y, por otra, evaluar su competencia pragmática, a partir del diseño de un sistema de evaluación basado en el mayor o menor uso de la atenuación. La metodología seguida para el análisis de la atenuación se basa en la propuesta de Albelda et al. (2014), en la que se tienen en cuenta variables situacionales, sociolingüísticas y enunciativas, así como una lista de posibles procedimientos candidatos a expresar las formas de atenuación. Los resultados del estudio dan cuenta, por un lado, de la necesidad social y comunicativa que personas de esta franja de edad tienen de justificarse al realizar un acto de habla de rechazo. Ello se observa a partir de la frecuencia de empleo y del tipo de mecanismos de atenuación. Por otro lado, evidencian la existencia de un porcentaje representativo de inadecuación por defecto y por exceso a la situación comunicativa, que implica un bajo nivel de adquisición de la competencia pragmática y que está representado mayoritariamente por el sexo masculino.

Palabras clave: atenuación, competencia pragmática, acto de habla de rechazo, WhatsApp.

ABSTRACT: In this paper, a study of pragmatic mitigation has been carried out from the analysis of a corpus of written samples that compile a simulation activity or role-play proposed to adolescent students (15-18 years old): the composition of a WhatsApp in which an invitation is rejected. The aim of this study is, on the one side, to observe the use that adolescents make of the pragmatic strategy of mitigation, and, on the other, to evaluate their pragmatic competence, from the design of an evaluation system based on the greater or lesser use of mitigation. The methodology followed so as to analyse mitigation is the one proposed by Albelda et al. (2014), in which situational, sociolinguistic and declarative 
variables are considered, as well as a list of possible procedures appointed to express mitigation forms. The results of the study state, on the one hand, the social and communicative need that people of this age group have to justify themselves when performing a refusal speech act. This is observed on the basis of the frequency of use and the type of mitigation procedures. On the other hand, the results show the existence of a representative percentage of inadequacy by default and by excess on the communicative situation, which implies a low acquisition level of pragmatic competence and which is mostly represented by males.

Keywords: mitigation, pragmatic competence, refusal speech act, WhatsApp.

\section{INTRODUCCIÓN}

En el presente trabajo asistimos a un estudio de la atenuación pragmática realizado a partir del análisis de un corpus de muestras escritas consistentes en una actividad de simulación o role-play. Dicha actividad ha sido elaborada por estudiantes de $4^{\circ}$ de la ESO, de entre 15 y 18 años y en ella han llevado a cabo la escritura de un WhatsApp en el que se rechaza a una invitación previa. Este análisis tiene un doble objetivo. Por una parte, se pretende observar el uso que hacen los adolescentes de esta estrategia pragmática a la hora de elaborar actos de habla de rechazo (respuestas despreferidas) dentro del género conversacional de WhatsApp, haciendo una elicitación de datos para determinar cuáles son las tácticas lingüísticas atenuantes a las que más se recurre en esta situación comunicativa concreta y por qué. Por otra parte, con el análisis de la atenuación, se pretende evaluar el nivel de competencia pragmática (Bachman, 1990) en personas de esta edad y en una situación concreta determinada. Comprobada la idoneidad de la atenuación como herramienta indicadora de la competencia pragmática en estudiantes (Magraner, en prensa), se ha diseñado un sistema de evaluación de la competencia pragmática basado en el mayor o menor uso de este fenómeno pragmático. En dicho sistema, que parte de las propuestas de Locher y Watts (2005) y Secchi (2017), se establecen tres niveles de adecuación pragmática: adecuado, inadecuado por defecto e inadecuado por exceso. De esta forma, el primer nivel se corresponde con una buena adecuación pragmática por el empleo de los mecanismos atenuantes suficientes y apropiados a la situación comunicativa concreta. El segundo nivel de adecuación se aplica a los casos en los que no se ha conseguido ser eficaz comunicativamente por no emplear (suficientes) mecanismos atenuantes. Como consecuencia de ello se aprecia una inadecuación a la situación comunicativa, que puede perjudicar la relación entre los interlocutores y la buena marcha del encuentro comunicativo. Finalmente, el tercer nivel se corresponde con aquellas actividades en las que vemos comportamientos hipercorteses (overpoliteness, Locher y Watts 2005), que, como indica Bernal (2007: 76), también son perjudiciales, ya que una cortesía exagerada puede mostrar una aplicación pragmática fallida. El sistema evaluativo propuesto ha sido evaluado a través de una encuesta a un grupo de informantes.

\section{MARCO TEÓRICO}

Los actos de rechazo a una invitación, por ser segundas partes despreferidas en un par adyacente, suelen expresarse acompañados de estrategias atenuantes. Este suele ser el comportamiento comunicativo esperado en un hablante competente pragmática y 
sociocomunicativamente. Atendiendo a la clasificación propuesta por Brown y Levison (1987), el rechazo constituye un acto de amenaza a la imagen (face treathening act), que, frente al acto de refuerzo a la imagen (face flattering act), "puede dañar o herir potencialmente la imagen de los demás» (Albelda y Barros, 2013: 13). Por tanto, para facilitar y mantener las relaciones sociales, será deseable restituir ese daño y, la atenuación, como mecanismo pragmático, puede ayudar considerablemente en este propósito. Como explican González y García (2017: 189):

El Análisis de la Conversación (AC) ha categorizado el rechazo como una respuesta despreferida dentro de los pares adyacentes en los que el primer miembro del par establece la relevancia de la aceptación o el rechazo (i.e., peticiones, invitaciones, etc.). Por definición, las respuestas despreferidas son más complejas y largas que las respuestas preferidas $[\ldots]$ por la aparición de mecanismos de indirección, tales como la tendencia a retardar la respuesta, la inclusión de excusas o justificaciones que acompañan al rechazo, y la atenuación interna del rechazo en sí.

Por su parte, la atenuación se considera como uno de los polos de la modulación discursiva (frente a la intensificación). En palabras de Haverkate, «podríamos definir el atenuante como una partícula, palabra o expresión que sirve para modificar el significado de un predicado de forma que se indique que ese significado solo se aplica parcialmente al objeto descrito» (1994: 209). La atenuación ha recibido en las últimas décadas una extensa atención, por considerarse una estrategia retórica de repercusión fundamental en la comunicación humana (Fraser, 1980; Meyer-Herman, 1988; Caffi, 1999). Para Caffi (1999: 883): «mitigation works in a multilayered and multi-dimensional way, simultaneously affecting a plurality of linguistic levels and interactional dimensions». Estos niveles o parámetros interaccionales son, para Caffi (1999) los argumentos de predicado: alguien atenúa algo a través de algún mecanismo. En otras palabras, se trata de un objeto lingüístico (el mecanismo lingüístico por el cual se mitiga) y de un componente abstracto del enunciado ${ }^{1}$, es decir, un rasgo del conglomerado de rasgos semántico-pragmáticos del enunciado. En este sentido, avanza una clasificación de los mecanismos atenuantes basada en los tres componentes del enunciado sobre los cuales la mitigación puede operar: la proposición, la ilocución y la fuente de la emisión. Extendiendo la metáfora de Lakoff (1973), les llama bushes, hedges y shields respectivamente.

Más recientemente, Briz y Albelda (2013) han destacado la dimensión estratégica de este fenómeno, al considerarla una categoría pragmática de naturaleza táctica e intencional, dirigida a la efectividad y la eficacia del discurso, y por tanto, al logro de los fines en la interacción. Así, de acuerdo con estos autores, se atenúa para lograr el acuerdo y la aceptación del otro interlocutor, para convencer, lograr un beneficio, persuadir y, a la vez, para cuidar las relaciones interpersonales y sociales. Sus fines son, por tanto, de carácter retórico pero también social. Así se aprecia en su propuesta de definición: «La atenuación es un mecanismo estratégico de distanciamiento lingüístico del mensaje y, a la vez, de acercamiento social: lingüísticamente, atenuación significa distancia; socialmente, atenuación significa acercamiento» (Briz y Albelda, 2013: 293).

Los mecanismos atenuantes pueden plasmarse en la lengua de dos formas: en la expresión del contenido proposicional (atenuación del dictum o intraproposicional) y en la expresión directa de la fuerza ilocutiva (atenuación del modus o de la enunciación) (Briz, 2005: 79). Por ejemplo, los diminutivos, los eufemismos o los extranjerismos, serían tácticas atenuantes del dictum, porque afectan al enunciado, es decir, repercuten

\footnotetext{
${ }^{1}$ Con mecanismos abstractos se refiere a la precisión de la referencia, al compromiso con la verdad de la proposición o a la intensidad de la fuerza ilocutiva, entre otros.
} 
sobre lo dicho. En cambio, mecanismos como la justificación, la disculpa o los emoticonos constituirían tácticas atenuantes del modus, porque afectan directamente a la enunciación, al decir.

Como hemos dicho, la atenuación es una estrategia y, por tanto, un mecanismo que contribuye a la negociación y al logro del acuerdo entre los interlocutores. Es, en este sentido también, un mecanismo retórico para convencer, persuadir y, a la vez, para evitar que las relaciones sociales se deterioren o sufran algún tipo de daño. Así, el uso adecuado de esta estrategia pragmática puede contribuir de forma muy significativa a que un determinado mensaje o enunciado sea pragmáticamente adecuado. Fijémonos en el siguiente ejemplo (1). Se trata de un mensaje extraído del corpus ${ }^{2}$, realizado por una alumna, en el que vemos diversos mecanismos atenuantes resaltados tipográficamente en negrita:

(1) Estebaaan! Que al final no voy al cine. La hora es muy tarde mi maama no me deja. Vamos otro dia?

Una prueba que manifiesta la relevancia de la atenuación en el logro de una competencia pragmática eficiente consiste en suprimir los mecanismos atenuantes del ejemplo anterior, como se puede ver en (1') a continuación:

(1') No voy al cine. La hora es muy tarde.

En (1') se aprecia un mensaje más directo, en el que únicamente se incorpora el contenido informativo que el emisor quiere transmitir a su interlocutor. Si bien se logra el traspaso de información, la eficiencia comunicativa de (1') resulta pobre, en tanto que no se activan todas las posibilidades que supone la competencia comunicativa. El dominio de la competencia gramatical y la corrección no son suficientes para una comunicación eficiente, es necesario que interactúen todas las subcompetencias que componen la comunicación. Así, en el caso de la subcompetencia pragmática, acudir a la atenuación puede resultar en una situación comunicativa como la planteada en (1) una forma de satisfacerla. La atenuación ayuda, por una parte, a conseguir el propósito comunicativo o fuerza ilocutiva (en este caso, rechazar una invitación o plan sin dañar la imagen y los sentimientos del otro) y, por otra, a reconocer las características del contexto de uso de la lengua para poder adecuarnos a ella. De ahí que, para el presente estudio, se haya acudido a la atenuación como fenómeno exponente para la evaluación de la competencia pragmática.

Como es sabido, la competencia pragmática se desarrolló a partir de la propuesta original de D. Hymes (1972). Entre otros modelos de descripción (Canale y Swain, 1980; Bachman, 1990; Celce-Murcia, Dörnyei y Thurrell, 1995), destaca la propuesta de Bachman (1990), por ser uno de los primeros en hablar de la competencia pragmática y en referirse a ella con ese nombre. Para este autor, la competencia comunicativa incluye las subcompetencias organizativa y pragmática. Dentro de la pragmática, distingue, a su vez, dos competencias más: la ilocutiva y la sociolingüística. Por una parte, la competencia ilocutiva se refiere a la relación entre los enunciados y actos o funciones que los hablantes o escritores intentan realizar a través de estos enunciados. En este sentido, la atenuación es un mecanismo que contribuye a desarrollar la competencia ilocutiva en tanto que ayuda a conseguir de forma eficiente el objetivo comunicativo. Por otra parte, la competencia sociolingüística determina el carácter adecuado de los enunciados en

\footnotetext{
${ }^{2}$ Todos los mensajes citados están transcritos literalmente tal y como los han realizado los adolescentes, incluyendo faltas de ortografía, de redacción, de puntuación y de estilo.
} 
función de las características del contexto de uso de la lengua. La atenuación también juega un papel determinante en esta competencia dado que es un mecanismo estratégico de acercamiento social a través del cual se cuidan las relaciones interpersonales.

Por tanto, la competencia pragmática podría definirse como «la habilidad de transmitir y comprender el intento comunicativo por medio de la ejecución e interpretación de los actos de habla y las funciones lingüísticas» (Celce-Murcia, Dornyei y Thurrell, 1995: 9). Para Bialystok (1993: 43), se describe del siguiente modo:

Pragmatic competence entails a variety of abilities concerned with the use and interpretation of language in contexts. It includes speakers' ability to use language for different purposes - to request, to instruct, to effect change. It includes listeners' ability to get past the language and understand the speaker's real intentions, especially when these intentions are not directly conveyed in the forms- indirect requests, irony and sarcasm are some examples.

En la evaluación de la competencia pragmática es necesario, junto a la observación del grado y tipo de atenuación empleada, analizar este fenómeno en relación con el contexto o situación comunicativa concreta en que se lleva a cabo el mensaje lingüístico. Como explica Magraner (en prensa): «Para la adquisición de la competencia pragmática $\mathrm{y}$, por tanto, para poder evaluarla en los estudiantes, es fundamental hacerlo juzgando cada uso lingüístico de acuerdo con su contexto y situación comunicativa». En principio, como señala Briz (2004), a mayor distancia, mayor frecuencia de atenuantes, mientras que a más proximidad social, menor uso de atenuación. La situación comunicativa que estudiamos en este trabajo, debería presentar pues pocos mecanismos atenuantes, por tratase de una situación informal, entre amigos. Sin embargo, la atenuación en ella es también necesaria, algo que podemos explicar a través del concepto de gravity propuesto por Brown y Levinson (1987), y que no contempla la clasificación de la variación situacional propuesta. Según estos autores, la carga amenazante de un acto de habla viene determinada por el cálculo de esta ecuación: FTAs $=\mathrm{D}$ (distance $)+\mathrm{P}($ power $)+\mathrm{G}$ (gravity). En este caso en concreto, tanto la distancia o relación vivencial de los interlocutores (D) como el poder o relación jerárquica existente entre ellos $(\mathrm{P})$, van en detracción del uso de la atenuación, ya que los interlocutores son dos amigos estudiantes. Pero la gravedad o peso del contenido semántico del mensaje emitido es lo que hace que se requieran mecanismos atenuantes. Por tanto, la gravedad, peso o coste del acto de habla sería lo que determinaría, en este caso, la presencia de atenuantes.

\section{METODOLOGÍA}

\subsection{CORPUS DE ANÁLISIS}

Para llevar a cabo el análisis, se ha creado un corpus propio, a través de la elicitación de datos en un ejercicio de expresión escrita por parte de adolescentes. Así, se les ha pedido que escribieran un mensaje de WhatsApp bajo una serie de instrucciones muy concretas en cuanto a la situación comunicativa: elaborar un acto de habla de rechazo para decirle a su mejor amigo/a, con el/la que han quedado para ir al cine, que al final no van a poder ir: 
Figura 1. Instrucción para la realización de la actividad

Actividad
Habías quedado con tu mejor amigo/a esta tarde para ir al cine, pero te ha salido un plan mejor
y no sabes cómo decírselo. Escríbele un WhatsApp y dile que finalmente no vas a poder
quedar con él/ella.

Como hemos dicho, la actividad constituye un acto de habla de rechazo y, como tal, se espera el empleo de mecanismos de indirección que ayuden a mitigar la amenaza que este implica. Entre tales mecanismos, la atenuación puede ocupar un papel primordial, al actuar como fenómeno mitigador de una potencial amenaza y al prevenir el daño o la intromisión en el territorio de la otra persona. Pero el uso de la atenuación que hagan los adolescentes ha de ser equilibrado y armónico, es decir, el adecuado a la situación propuesta, ya que, tanto los comportamientos hipercorteses como los insuficientes en el recurso a este fenómeno, serán evaluados como inadecuados.

Seguidamente, se detallarán las características de los informantes y del corpus son las siguientes. Las muestras se obtuvieron durante el segundo trimestre (enero-marzo) del curso escolar 2017-2018, en el IES Districte Marítim de la ciudad de Valencia. Un total de 44 estudiantes de $4^{\circ}$ de la ESO pertenecientes a tres grupos distintos realizaron la actividad explicada, a los cuales se les requerían, además, los siguientes datos: nombre, apellidos, fecha de nacimiento, curso, grupo, lengua materna y nacionalidad. Esto nos han permitido establecer distintas variables como el sexo, la edad, la lengua materna o la nacionalidad, que nos han ayudado considerablemente a comprender las actividades de cada alumno y a evaluarlas:

\section{Tabla 1. Variables del corpus de análisis}

\begin{tabular}{|c|c|c|}
\hline \multicolumn{3}{|c|}{ VARIABLES } \\
\hline \multirow{2}{*}{ Sexo } & Mujer: 23 & \multirow{15}{*}{ Total: 44} \\
\hline & Hombre: 21 & \\
\hline \multirow{3}{*}{ Edad } & 2000 (17-18 años): 5 & \\
\hline & 2001 (16-17 años): 16 & \\
\hline & 2002 (15-16 años): 23 & \\
\hline \multirow{3}{*}{ Lengua materna } & Valenciano: 2 & \\
\hline & Castellano: 39 & \\
\hline & Valenciano/castellano: 3 & \\
\hline \multirow{7}{*}{ Nacionalidad } & Español/a: 36 & \\
\hline & Colombiano/a: 2 & \\
\hline & Ecuatoriano/a: 1 & \\
\hline & Dominicano/a: 1 & \\
\hline & Ecuatoriano/a-Español/a: 2 & \\
\hline & Colombiano/a-Español/a: 1 & \\
\hline & Británico/a-Español/a: 1 & \\
\hline
\end{tabular}

En definitiva, se ha trabajado con un corpus escrito en español, formado por 44 mensajes, escritos por 44 adolescentes ( 23 chicas y 21 chicos) residentes en la ciudad de Valencia, de entre 15 y 18 años. 


\subsection{PARÁMETROS DE ANÁLISIS}

\subsubsection{Parámetros para el análisis de la atenuación pragmática}

El análisis de un fenómeno pragmático, como es el de la atenuación en muestras discursivas, requiere de un sistema objetivo de reconocimiento de tal función comunicativa en las formas lingüísticas. Para ello, es necesario contar con una metodología que nos permita asegurar, como investigadores, que el hablante en su intención original pretendió ser atenuado al emplear determinadas formas lingüísticas. Así, hemos acudido a una propuesta metodológica de análisis de la atenuación (Albelda et al., 2014), que ha servido de base a diversos estudios de naturaleza pragmática. Esta propuesta incluye parámetros situacionales, sociolingüísticos y enunciativos, así como una lista de posibles procedimientos candidatos a expresar las formas de atenuación, lo que permite tener en cuenta todas las posibles influencias contextuales extralingüísticas que puedan motivar y presionar el empleo de determinadas formas lingüísticas. Pero el hecho de que la ficha esté diseñada y pensada para trabajar con corpus orales ha hecho que tengamos que introducir unas pequeñas modificaciones. Las tácticas atenuantes que se proponen en este trabajo para el análisis del género escrito del WhatsApp son las siguientes:

1. Modificadores morfológicos internos: sufijos (diminutivos).

2. Modificadores externos: cuantificadores minimizadores, aproximativos o difusores significativos proposicionales o extraproposicionales.

3. Términos o expresiones más suaves en el contenido significativo.

4. Empleo de palabras extranjeras.

5. Usos modalizadores (dislocados) de los tiempos verbales.

6. Empleo de verbos, construcciones verbales, así como de partículas discursivas con valor modal que expresan opiniones en forma de duda o probabilidad.

7. Empleo de verbos, construcciones verbales y partículas discursivas que expresan fingimiento de incertidumbre, de incompetencia o de ignorancia.

8. Peticiones, preguntas, mandatos u órdenes expresados de forma indirecta.

9. Expresiones de disculpa.

10. Estructuras sintácticas o significados condicionales, concesivos o temporales, que modifican y restringen el alcance del acto de habla.

11. Partículas y construcciones justificadoras o de excusa.

12. Impersonalizaciones (I).

13. Movimientos de reformulación.

14. Concesividad.

15. Partículas discursivas evidenciales.

16. Partículas discursivas y expresiones de control de contacto.

17. Uso de formas de tratamiento y de fórmulas apelativas convencionalizadas ${ }^{3}$.

18. Empleo de elementos prosódicos, paralingüísticos o gestuales ${ }^{4}$.

\footnotetext{
${ }^{3}$ También se incluyen aquí las fórmulas apelativas convencionalizadas anticorteses. La anticortesía queda definida por Albelda y Barros (2013: 25) de la siguiente forma: «Denominaremos, por tanto, anticorteses, a aquellos actos de habla que emplean formas y procedimientos descorteses con los que se ataca la imagen del otro, pero en los que el contexto neutraliza la potencial ofensa».

${ }^{4}$ El motivo por el que hemos decidido conservar esta táctica propia de la oralidad en un corpus escrito es que los alumnos, en un espacio comunicativo como el WhatsApp, intentan imitar frecuentemente la oralidad. Así, podemos ver esta táctica adaptada a la escritura a través de las risas (jajaja, jejeje) -elemento paralingüístico-, del alargamiento de determinadas vocales (tiooo) -elemento prosódico- y de los
} 
19. Palabras o expresiones cuyo contenido semántico expresa solidaridad y empatía con el interlocutor al que se dirige.

20. Ironía.

21. Expresión de sentimientos con la finalidad de minimizar o reparar el daño hecho.

22. Emoticonos.

23. Recompensas.

En tanto que este corpus de análisis ha generado un tipo particular de acto de habla, el rechazo a una invitación, la gestión de la atenuación en estos casos parece que se especializa en una serie de tácticas lingüísticas, algunas de las cuales no se encuentran en la ficha de análisis mencionada. El rechazo, como se ha visto, constituye un tipo de respuesta despreferida, y como tal, esta es más compleja y más larga que la respuesta preferida. Así pues, se han introducido cinco nuevas tácticas lingüísticas de atenuación, que, aunque no son intrínsecamente atenuantes, sí lo son en ese contexto concreto del rechazo: la expresión de empatía, la ironía, la expresión de sentimientos (te quiero, te amo), los emoticonos y la recompensa, tácticas que ocupan, respectivamente, los números 19, 20, 21, 22 y 23 en el listado mencionado anteriormente. La inclusión de estas tácticas lingüísticas atenuantes no contempladas en la ficha original ha sido posible gracias al análisis cualitativo de cada acto de habla de rechazo, en el que se ha tenido en cuenta el contexto interactivo y situacional concreto. En definitiva, se han realizado algunas modificaciones en la ficha de ES.VAR.ATENUACIÓN que, sin alterar su estructura básica, complementarán esta herramienta metodológica y ayudarán a realizar una descripción más precisa de la atenuación en el género escrito manejado en el corpus, el WhatsApp.

\subsubsection{Sistema de evaluación de la competencia pragmática}

Una vez realizado el estudio de la atenuación pragmática, hemos evaluado la adecuación pragmática de cada una de las respuestas de los participantes en la simulación. En ese sentido, como explica Briz (1998: 25): «La falta de adecuación entre el uso y la situación provocaría desajustes no tanto informativos como de conducta lingüística esperable». Esta inadecuación pragmática puede resolverse y mejorarse en estos casos a través del uso de la estrategia de la atenuación, ya que esta puede ayudarnos a evitar posibles desajustes comunicativos que tienen repercusión en las relaciones sociales entre los interlocutores. Se ha diseñado, pues, un sistema de evaluación de la competencia pragmática compuesto por tres niveles de adecuación: adecuado, inadecuado por defecto e inadecuado por exceso. Este sistema parte del modelo propuesto por Secchi (2017) y también de los diferentes tipos de relational work establecidos por Locher y Watts (2005). Las correspondencias entre los tipos de trabajo relacional de Locher y Watts (para el estudio de la cortesía verbal) y los tres niveles aquí considerados es la siguiente:

emoticonos, a través de los cuales podemos deducir algunos de los gestos que los adolescentes harían si oralizasen la conversación. 
Tabla 2. Relación entre los tipos de relational work de Locher y Watts (2005) y el sistema de evaluación de la competencia pragmática

\begin{tabular}{|c|c|}
\hline Niveles de adecuación pragmática & $\begin{array}{l}\text { Relational Works (Locher y Watts, } \\
\text { 2005) }\end{array}$ \\
\hline$\frac{\text { Nivel } 1}{\text { Adecuado }}$ & $\begin{array}{l}\text { Relational work } 3 \\
\text { Marcada positivamente, cortés, apropiada } \\
\text { políticamente. }\end{array}$ \\
\hline$\frac{\text { Nivel } 2}{\text { Inadecuado por defecto }}$ & $\begin{array}{l}\text { Relational work } 1 \\
\text { Marcada negativamente, descortés, no } \\
\text { apropiada políticamente. } \\
\text { Relational work } 2 \\
\text { No marcada, neutra, acortés (ni cortés ni } \\
\text { descortés, normal), apropiada } \\
\text { políticamente. }\end{array}$ \\
\hline$\frac{\text { Nivel } 3}{\text { Inadecuado por exceso }}$ & $\begin{array}{l}\text { Relational work } 4 \\
\text { Marcada negativamente, sobrecortés } \\
\text { (overpolite), no apropiada políticamente. }\end{array}$ \\
\hline
\end{tabular}

Así pues, se han analizado todas las muestras del corpus de acuerdo con este sistema evaluativo con el objetivo de ver si los adolescentes tienen desarrollada la competencia pragmática en el género del WhatsApp y si son capaces de adecuarse a la situación comunicativa que se les presenta. Los tres niveles de adecuación pragmática propuestos se justifican de la siguiente manera:

1. Adecuado: el/la alumno/a se adecúa perfectamente a la situación comunicativa concreta que se le propone, en tanto que ha empleado las estrategias de atenuación apropiadas y necesarias.

2. Inadecuado por defecto: el/la alumno/a no se adecúa a la situación comunicativa concreta que se le propone, en tanto que no ha empleado (suficientes) estrategias de atenuación.

3. Inadecuado por exceso: el/la alumno/a no se adecúa a la situación comunicativa concreta que se le propone, en tanto que ha empleado demasiadas estrategias de atenuación (overpoliteness).

Asimismo, con el fin de objetivar este sistema evaluativo de la competencia pragmática propuesto, se ha realizado una validación que permita asegurar que otros hablantes de la lengua la juzguen en los mismos términos de (in)adecuación. Para dicha validación se ha realizado una encuesta a un grupo de informantes (15 adolescentes y 15 adultos). Así, se les ha pasado la misma prueba que al grupo de adolescentes de nuestro corpus junto con tres de las respuestas de dichos jóvenes. Las respuestas son las siguientes:

Figura 2. Respuesta 1 de la encuesta de validación del sistema evaluativo de la competencia pragmática.

Hola Pepa!!! Soy Laia! Lo siento pero esta tarde me es imposible quedar. No me acordaba de que tenía médico a las 17:00. Ojalá poder qedar otro día. Sorry $-\approx \mathrm{Tq}$ 
Figura 3. Respuesta 2 de la encuesta de validación del sistema evaluativo de la competencia pragmática.

- Eh pringao, que no puedo quedar.

- Tengo que vacunarme de la alergia.

Figura 4: Respuesta 3 de la encuesta de validación del sistema evaluativo de la competencia pragmática

Rafa, tiio, me ha salido una cena con los del cole, justo hoy se han puesto todos de acuerdo para irnos por ahí a cenar, pff tío, lo siento muchísimo.

Cancelamos el cine, ¿vale?, dime si la semana que viene puedes quedar para ir y si esta la misma peli la vemos, hasta te invitaré a las palomitas, lo siento mucho eh, enserio, venga tío, nos vemos.

Así, se les ha pedido que valoraran dichas respuestas en función de tres posibles juicios pragmáticos: adecuado, inadecuado por defecto o inadecuado por exceso. A continuación, se presentan los resultados de esta encuesta:

Figura 5. Resultados de validación del sistema evaluativo de la competencia pragmática

RESULTADOS RESPUESTA 1

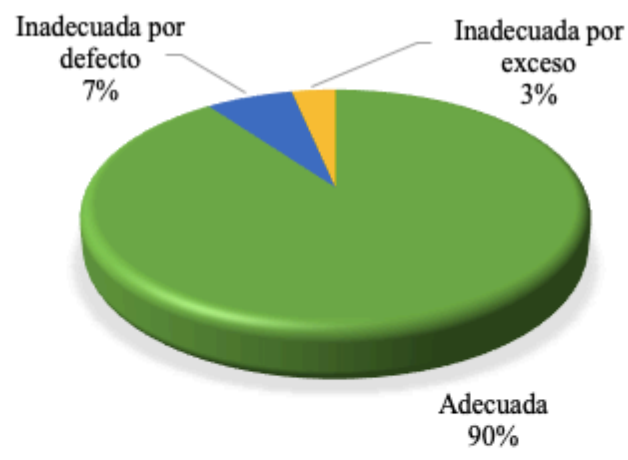

RESULTADOS RESPUESTA 2

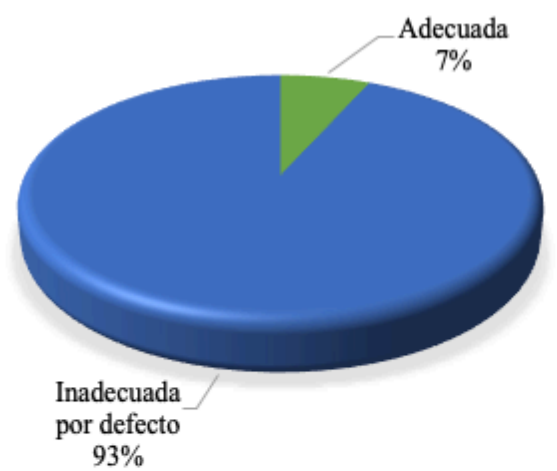

RESULTADOS RESPUESTA 3

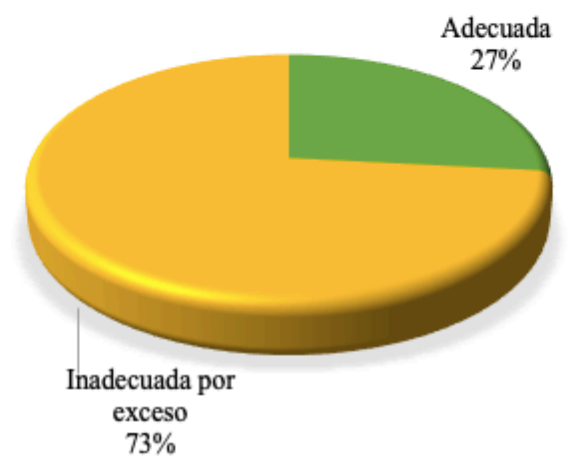


Como se puede ver, los informantes contestan, en general, en un $85,33 \%$ de las ocasiones de acuerdo al criterio establecido para elaborar el sistema de evaluación, es decir, en un $90 \%$ de los casos, los informantes consideran que la respuesta 1 es adecuada, en un $93 \%$, que la respuesta 2 es inadecuada por defecto y en un $73 \%$, en que la tercera respuesta es inadecuada por exceso. Por tanto, los resultados de la encuesta avalan y validan este sistema evaluativo.

\section{RESULTADOS DEL ANÁLISIS}

En este apartado se presentan los resultados del análisis del corpus, organizado en dos bloques o apartados. En el apartado 4.1. se exponen los resultados del análisis de los mecanismos atenuantes utilizados por los adolescentes y en el 4.2, se muestran los resultados del análisis de la evaluación de la competencia pragmática, que son, en ambos casos, tanto cuantitativos como cualitativos.

\subsection{RESULTADOS DEL ANÁLISIS DE LAS TÁCTICAS LINGÜÍSTICAS DE ATENUACIÓN}

Tras el análisis de los 44 mensajes, se han identificado 302 tácticas lingüísticas de atenuación. A continuación, se presentan los resultados de dicho análisis y se señala el porcentaje que representa dentro del corpus analizado cada una de las tácticas lingüísticas:

Figura 6. Tácticas lingüísticas de atenuación: resultados generales

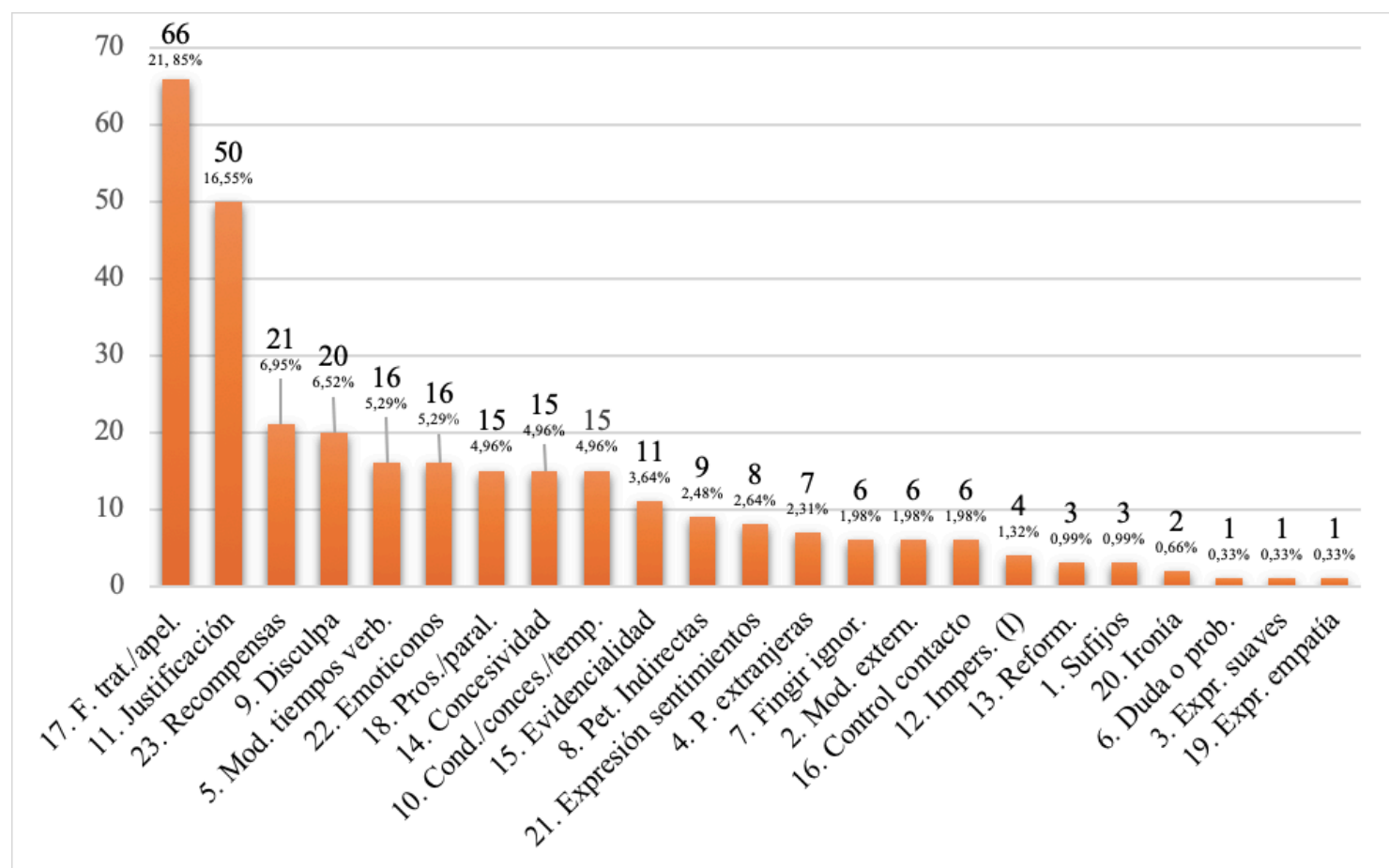

La Figura 6 muestra que la estrategia lingüística de atenuación más empleada por los adolescentes son las formas de tratamiento (nombres propios) y las fórmulas apelativas convencionalizadas (del tipo tío/a, hombre/mujer, venga, va, mira, escucha), con un $21,85 \%$ de representación en el corpus. A través de este mecanismo consiguen mostrar acercamiento hacia el otro y minimizar lo dicho en tanto amenaza para este. En concreto, 
dentro del acto de habla de rechazo, los adolescentes recurren a muchas y diferentes fórmulas apelativas convencionalizadas para atenuar, entre las que destaca la de tío/tía, como vemos en el ejemplo (2):

(2) Tía, Carmen, no voy a poder quedar porque tengo médico, nos vemos otro día. Sorry $<3$

Mañana te cuento.

$14 \mathrm{MCE}^{5}$

También se incluyen aquí las fórmulas apelativas convencionalizadas anticorteses, puesto que, a pesar de ser procedimientos descorteses, el contexto consigue neutralizarlas y acaban mostrando un acercamiento hacia el otro. Algunas de estas fórmulas las podemos ver en los siguientes ejemplos:

(3) - Eh pringao,

que no puedo

quedar.

- Tengo que vacunarme

de la alergia.

4HCE

(4) Eh tú, desgraciao’,

No puedo ir al cine

Tengo que irme al

centro, he quedado para coger un tren

$5 \mathrm{MCE}$

Otro procedimiento de atenuación muy frecuente en el corpus es el de las partículas y construcciones justificadoras o de excusa, con un 16,55\% de representación. Para minimizar la amenaza que implica elaborar un acto de habla de rechazo, en este caso en concreto, los adolescentes recurren a la estrategia de la justificación, que, en la mayoría de los casos se trata de partículas como es que, porque, como, que [causal], lo que pasa es que, etc. En el siguiente ejemplo vemos claramente el protagonismo que esta táctica lingüística de atenuación ha tenido en el corpus:

(5) Escuchame Basti que esta tarde al final no puedo ir a ver Lluvia de albondigas porque me ha llamado mi madre para que le ayude a hacer unas faenas si acabo pronto te aviso pero no creo que tarde poco así que ves haciendo planes y sí eso te aviso lo siento bro pero esque sino no me dejan salir el viernes.

2HEE

En el ejemplo (5) vemos tres partículas justificadoras o de excusa: al final, porque y esque. Al final sería una partícula de excusa con un matiz temporal con la que el informante indica que el hecho de que no poder ir queda justificado por haber tenido un imprevisto. También se han enmarcado partículas como porque, ya que, por o dentro de la justificación porque a través de ellas los adolescentes introducen las justificaciones o explicaciones posteriores que avalan por qué han actuado o están actuando de ese modo.

\footnotetext{
${ }^{5}$ Cada uno de los ejercicios está etiquetado de esta forma, cuyos caracteres representan lo siguiente: el 14 indica que es la decimocuarta alumna del grupo; la $\mathrm{M}$ indica el sexo, mujer; la $\mathrm{C}$, el grupo; y la $\mathrm{E}$, la nacionalidad, española.
} 
Con un grado también alto de empleo se sitúan las recompensas y los mecanismos de disculpa, que representan un $6,95 \%$ y un $6,62 \%$ del corpus, respectivamente. En tanto que se trata de un acto de habla de rechazo, tienen que recompensar de alguna forma la amenaza a la imagen del otro y disculparse por el posible daño causado. De ahí que estas sean dos estrategias bastante recurrentes en el corpus, como podemos ver en el ejemplo (6):

(6) Rafa, tiio, me ha salido una cena con los del cole, justo hoy se han puesto todos de acuerdo para irnos por ahí a cenar, pff tío, lo siento muchísimo.

Cancelamos el cine, ¿vale?, dime si la semana que viene puedes quedar para ir y si esta la misma peli la vemos, hasta te invitaré a las palomitas, lo siento mucho eh, enserio, venga tío, nos vemos.

8HEE

Son movimientos estratégicos relativamente frecuentes los modalizadores (dislocados) de los tiempos verbales (5,29\%), los cuales suponen una desfocalización del eje temporal, y, por ello, expresan la acción de forma más suave. Con el mismo número de ocurrencias (16) encontramos el mecanismo de los emoticonos (5,29\%), que, como indica Sampietro (2016: 274): «se asociarían [...] a actos expresivos y podrían contribuir a crear afiliación entre los interlocutores». En el ejemplo (7), podemos ver ambas tácticas en un mismo mensaje:

(7) Eyy!

Tia, esta tarde no voy a poder quedar contigo, mi madre me ha dicho que tengo que acompañarle al médico, y resulta que yo también tengo cita con él.

Ya sabes lo mucho que quería quedar contigo esta tarde, porque te echo mucho de menos y estaba ansiosa por ver esa película.

Lo siento, no te enfades conmigo.

Te quiero!

12MCE

Aquí la informante sustituye el presente (puedo) por una perífrasis verbal de futuro próximo (voy a poder), y el presente (quiero) por el condicional (quería) consiguiendo de esta forma hacer una desfocalización del eje temporal que expresa la acción de una forma más suave. Además, también se observa el emoticono del corazón, el cual ha sido muy recurrente en el corpus, especialmente por parte de las chicas. Pero también es destacable el uso de los emoticonos que expresan sentimientos o estados de ánimo en forma de carita, los cuales utilizan para acercarse al otro y mostrar empatía hacia él:

(8) Eh tú, desgraciao,

No puedo ir al cine

Tengo que irme al

centro, he quedado para coger un tren

Me voy con la cámara y los del pueblo

Intentaré no matarme en el Urbex

Lo siento, disfrutad sin mí

Por último, las tácticas menos utilizadas, con apenas representatividad en el corpus, son la ironía, con un 0,66\%; los términos o expresiones más suaves en el contenido significativo; el empleo de verbos, construcciones verbales y partículas discursivas con valor modal que expresan opiniones en forma de duda o probabilidad; $y$ las palabras o expresiones cuyo contenido semántico expresa solidaridad y empatía con 
el interlocutor al que se dirige, representando cada una de estas tres tácticas solo el 0,33\% del corpus. Estos serían pues los mecanismos lingüísticos menos usados por los adolescentes en el acto de habla de rechazo dentro del género conversacional de WhatsApp de la muestra recogida.

Veamos ahora también la comparación de las tácticas lingüísticas de atenuación en cada uno de los dos sexos con el objetivo de ver si se emplean de distinta manera. Los resultados de dicho análisis son los siguientes:

\section{Figura 7. Tácticas lingüísticas de atenuación: resultados en la comparativa chicos-chicas}

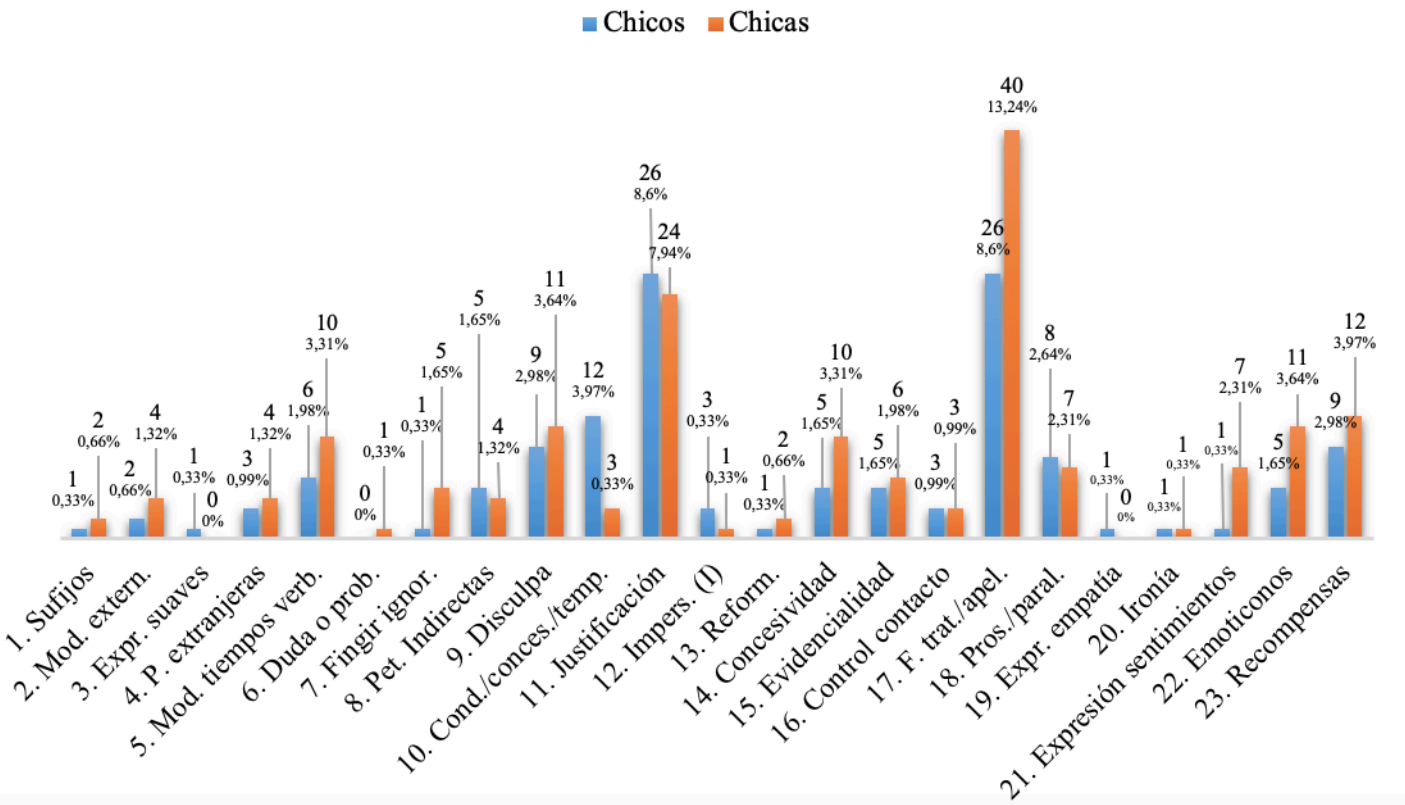

En general, la diferencia entre chicas $(168-55,62 \%)$ y chicos $(134-44,37 \%)$ en el uso de tácticas atenuantes no es muy grande, y más teniendo en cuenta que hay dos informantes más del sexo femenino (23) que del masculino (21). Pero, si se profundiza en los resultados obtenidos, se observa que hay algunas diferencias notables entre ambos sexos. En primer lugar, las tácticas más utilizadas por los chicos son la justificación (ver ejemplo 4), y las formas de tratamiento y fórmulas apelativas convencionalizadas (ver ejemplo 3), con un $8,6 \%$ de representación cada una en el corpus ${ }^{6}$. En el caso de las chicas también ha sido la justificación la estrategia atenuante más empleada (ver ejemplo 2) pero la diferencia entre ambos sexos es notable, ya que ellas la han usado hasta en un 13,24\% de las ocasiones. En segundo lugar, mientras que la táctica no utilizada por los chicos ha sido la de los verbos, construcciones verbales, y partículas discursivas con valor modal que expresan opiniones en forma de duda o de probabilidad, las chicas no han hecho uso de términos o expresiones más suaves en el contenido significativo (lítotes, eufemismos) ni tampoco de palabras o expresiones cuyo contenido semántico expresa solidaridad y empatía con el interlocutor.

Finalmente resulta interesante señalar las principales diferencias entre los dos sexos en lo que respecta al uso de la atenuación. La primera táctica en la que vemos una diferencia notable es la de la expresión de sentimientos, ya que, mientras que las chicas

\footnotetext{
${ }^{6}$ Todos los porcentajes expresados en la comparativa centrada en la variable sexo hacen referencia al total del corpus.
} 
la utilizan hasta en 7 ocasiones $(2,31 \%)$, los chicos, solo una $(0,33 \%)$. Esto constata que las chicas tienden a abrirse y a expresar más sus sentimientos hacia el otro que los chicos. La segunda táctica a comentar sería la de los emoticonos, ya que mientras que en las chicas se observan 11 ocurrencias $(3,64 \%)$, en los chicos solo $5(1,65 \%)$. Además, en el caso de los adolescentes, suelen ser emoticonos menos expresivos, como el del brazo fuerte o el del aplauso, que los que utilizan las adolescentes que, en su gran mayoría, son corazones. La tercera táctica con diferencia de uso es la de las estructuras sintácticas o significados condicionales (si te parece), concesivos (aunque sea tarde) o temporales (cuando te venga bien), que modifican y restringen el alcance del acto de habla, ya que los chicos la utilizan en 12 ocasiones $(3,97 \%)$ y las chicas solo en $3(0,99 \%)$. Además, esta táctica es destacable en la medida en la que es una de las pocas en las que predomina el uso de los chicos sobre el de las chicas. Por último, es destacable también la táctica de los verbos, construcciones verbales y partículas discursivas que expresan fingimiento de incertidumbre, de incompetencia o de ignorancia, ya que las chicas recurren a ella en 5 ocasiones $(1,65 \%)$ y ellos solo en una $(0,33 \%)$.

\subsection{RESUlTAdOS DE LA EVALUACIÓN DE LA COMPETENCIA PRAGMÁTICA}

Veamos ahora los resultados del análisis del corpus en lo relativo a la (in)adecuación pragmática en el uso de la atenuación. Siguiendo el sistema evaluativo presentado en la metodología e integrado por tres niveles de adecuación pragmática, hemos determinado si los mensajes eran o no adecuadas pragmáticamente. En la Figura 8 se recogen los porcentajes que representan dentro del corpus cada uno de los niveles de adecuación pragmática:

\section{Figura 8. Resultados generales de la evaluación de la competencia pragmática}

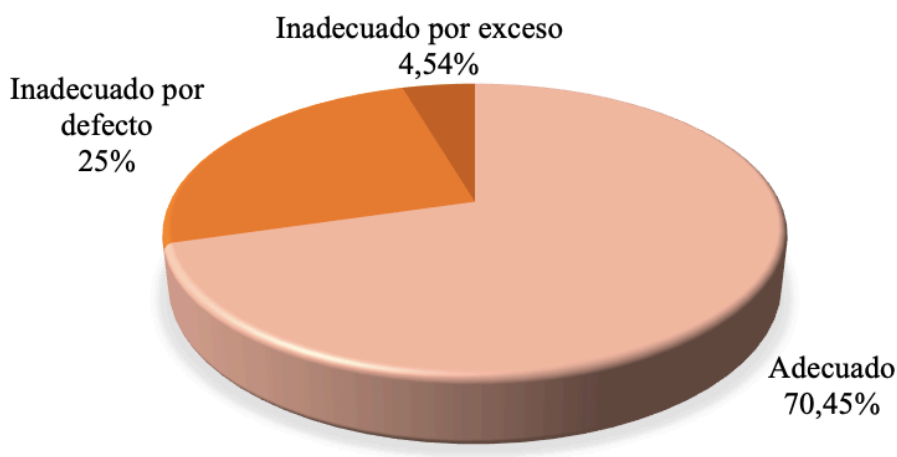

La Figura 8 muestra cómo en la mayoría de los casos (70,45\%) los alumnos han sabido adecuarse a la situación comunicativa propuesta, haciendo un buen uso de la competencia pragmática. En el siguiente ejemplo podemos ver una muestra de un caso en que se ha juzgado como excelente la adecuación pragmática por parte del alumno en su mensaje:

(8) Tio al final no puedo ir, lo siento pero te juro que va enserio, me es imposible, tengo que cuidar de mi hermano que esta con 39 de fiebre y mi madre esta trabajando, si quieres quedamos el viernes y te invito si quieres pero es que hoy es imposible $:$... No te piques 
En el ejemplo (8) vemos cómo el alumno se adecúa muy bien pragmáticamente no solo por emplear las estrategias de atenuación apropiadas y necesarias para la situación comunicativa concreta que se le ha presentado, sino también por representar a través de dibujos y símbolos (bocadillo verde, doble tic azul, hora de envío) el mensaje en el ciberespacio que se le requería, el WhatsApp. Sin embargo, casi el 30\% de los mensajes han sido inadecuados, ya sea por defecto $(25 \%)$ o por exceso $(4,54 \%)$. Este resultado pone de manifiesto que, a pesar de que el WhatsApp es un género que los jóvenes usan a diario, no todos saben resolver a través de él un acto de habla de rechazo de forma pragmáticamente eficiente. Asimismo, ha de hacerse notar que, incluso los mensajes que han sido considerados adecuados, podrían mejorarse a través del empleo de la atenuación, lo que les permitiría desempeñar un mayor uso retórico de la lengua y asegurarse que los destinatarios reciben los mensajes con mayor aceptación. Esta inadecuación la podemos ver en mensajes como el siguiente:

(9) Estebaaan! Que al final no voy al cine. La hora es muy tarde mi maama no me deja. Vamos otro dia?

$16 \mathrm{HCE}$

En este mensaje el alumno no se adecúa por no emplear suficientes estrategias de atenuación, ya que no aprecia plenamente que se enfrenta a una situación delicada en la que tiene que rechazar un plan con el que se había comprometido y que este requiere ser atenuado. Así, en la mayor parte del texto, el adolescente no demuestra implicación o empatía emocional con la persona a la que se dirige y con el mensaje que transmite. Una justificación más elaborada y el uso de mecanismos atenuantes como la disculpa o los emoticonos, podrían haber suavizado la transmisión de esta información. Pero la inadecuación no viene siempre de la mano de la escasez, sino que también puede estar originada por el exceso, como vemos en (10):

(10) - Hola Cristina

- ye parcera no voy a poder quedar con vos.

- esque un familiar se enfermo y esta en el hospital y pues estoy muy preocupada por el y pues tengo que ir a visitarlo, espero que me entiendas.

- pero otro dia quedamos, cuando usted quiera me dice yo le caigo, no se valla a enojar conmigo pana, entendeme

- estamos hablando parcerita, perdoneme jajaja

$8 \mathrm{MFC}$

En este caso el mensaje es inadecuado porque la alumna emplea demasiadas estrategias de atenuación para la situación comunicativa concreta que se le ha presentado. Se esfuerza en exceso por intentar justificar su rechazo y, además, pide disculpas o comprensión hasta en cuatro ocasiones distintas: espero que me entiendas, no se valla a enojar conmigo, entendeme, perdoneme, algo innecesario porque su no asistencia ya queda suficientemente justificada previamente. De esta forma, debido a un exceso de atenuación, muestra una aplicación pragmática fallida o errónea. Es importante señalar que la informante, en este caso, es colombiana, y que, por tanto, esta hipercortesía podría deberse a cuestiones culturales, ya que, «el español de muchas zonas de América, de acuerdo con los datos facilitados por algunos estudios empíricos, es, en general, más atenuado que el español europeo» (Briz y Albelda, 2013: 248).

También resulta interesante en este caso observar la variable del sexo, es decir, observar cuál de los dos grupos de informantes, las chicas o los chicos, se han adecuado 
mejor a la situación comunicativa, ya que la diferencia es muy notoria. Las Figuras 9 y 10 recogen los resultados del análisis de la adecuación pragmática de cada uno de los sexos de forma comparativa:

\section{Figuras 9 y 10. Competencia pragmática chicos y competencia pragmática chicas, respectivamente}
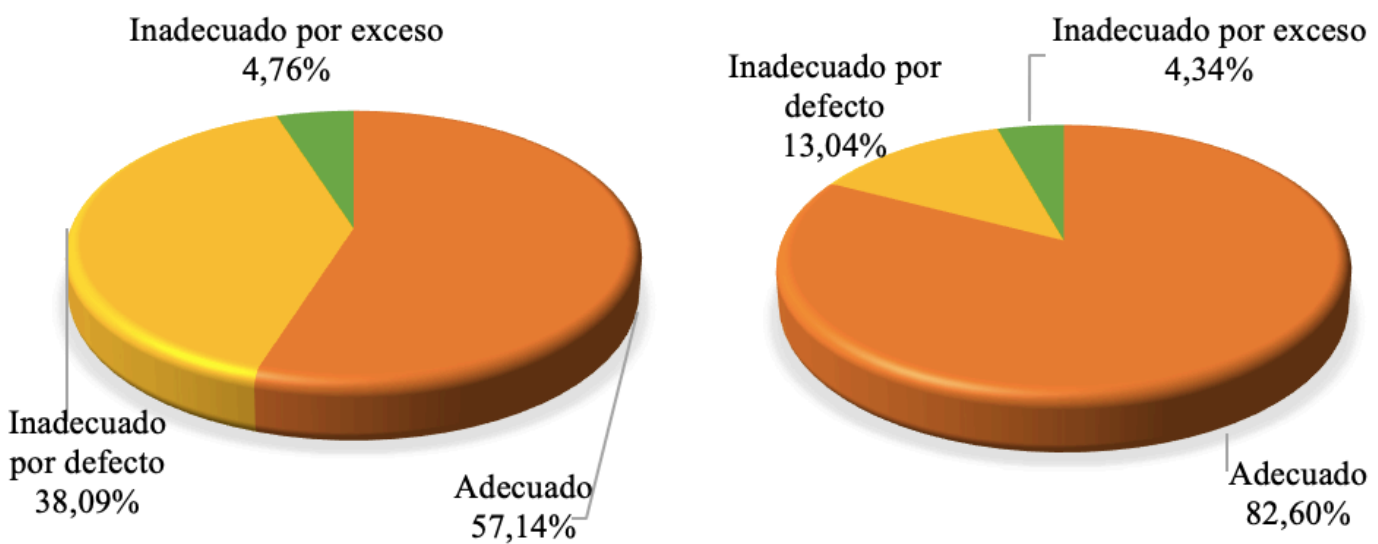

Como se puede observar, las diferencias son notables, ya que, mientras que las chicas han sabido adecuarse a la situación comunicativa en un $82,60 \%$ de los casos, los chicos solo lo han hecho en un $57,14 \%$ de las muestras. La inadecuación que vemos en los chicos se debe a la falta de tácticas lingüísticas de atenuación, ya que, en el 38,09\% de los mensajes, los adolescentes no han sido pragmáticamente eficientes debido a la no utilización de (suficientes) mecanismos atenuantes. Esto queda avalado por los resultados de la atenuación en la comparativa chicos-chicas, en la cual ellos hacen uso de 134 tácticas atenuantes frente a las 168 de ellas. Por otra parte, la inadecuación por exceso ha sido más esporádica, ya que solo se ha dado aproximadamente en un $5 \%$ de los mensajes tanto de chicos como de chicas.

\section{CONCLUSIONES}

A través de este estudio se ha pretendido analizar, en primer lugar, el mecanismo pragmático de la atenuación en actos de habla de rechazo en adolecentes dentro del género conversacional de WhatsApp. La elicitación de datos sobre una actividad de role-play ha permitido efectuar dicho análisis, cuyos resultados son los siguientes. En primer lugar, las tácticas a las que más han recurrido los adolescentes de la muestra recogida han sido las formas de tratamiento (nombres propios) y fórmulas apelativas convencionalizadas (especialmente la de $t i o / a$ ), con un $21,85 \%$ de representación en el corpus, entre las que destacan también las fórmulas apelativas anticorteses (pringao, desgraciao, tonta, etc.); y la justificación, con un 16,55\%. En segundo lugar, los resultados también nos han permitido saber que los adolescentes no utilizan la ironía, los eufemismos o lítotes, no expresan duda o probabilidad ni tampoco muestran empatía para atenuar los actos de habla de rechazo en el género del WhatsApp, pues todos estos mecanismos apenas tienen representatividad en el corpus analizado. Por otra parte, el estudio ha mostrado que son las chicas las que tienden a abrirse y a expresar más sus sentimientos hacia el otro $(2,31 \%)$. Solo un chico en todo el corpus analizado recurre a esta táctica $(0,33 \%)$. Asimismo, también las mujeres recurren más a los emoticonos como estrategias para atenuar sus actos de habla ( $3,64 \%$ chicas $-1,65 \%$ chicos $)$, mientras que los chicos utilizan 
más estructuras sintácticas o significados condicionales, concesivos o temporales $(3,97 \%)$ que las chicas $(0,99 \%)$.

El segundo objetivo de esta investigación ha sido evaluar la competencia pragmática de los adolescentes gracias al análisis de la mencionada atenuación lingüística, por ser este un mecanismo de naturaleza pragmática que puede ayudar a mejorar de forma notable la eficiencia comunicativa y las relaciones interpersonales. Así, se ha evaluado la adecuación en el uso de este mecanismo en adolescentes a través de un sistema basado en el mayor o menor uso de la atenuación en el que se establecen tres niveles de adecuación pragmática: adecuado, inadecuado por defecto e inadecuado por exceso (basado parcialmente en Secchi, 2017 y en Locher y Watts, 2005). Los resultados de dicho análisis son los siguientes: en la mayoría de los casos $(70,45 \%)$ los alumnos han sabido adecuarse a la situación comunicativa, haciendo un buen uso de la competencia pragmática. Sin embargo, casi el 30\% de los mensajes han sido inadecuados, ya sea por defecto $(25 \%)$ o por exceso $(4,54 \%)$. Este resultado pone de manifiesto que, a pesar de que, en general, la competencia pragmática mostrada es buena, no todos los adolescentes saben resolver a través del WhatsApp, un género que los jóvenes usan a diario, un acto de habla de rechazo de forma pragmáticamente eficiente. El análisis comparativo basado en la variable sexo, también ha revelado que mientras que las chicas han sido adecuadas pragmáticamente en un $82,60 \%$ de los casos, los chicos solo lo han sido en un $57,14 \%$ de los casos, ya que en el 38,09\% de los mensajes no han utilizado (suficientes) mecanismos atenuantes.

Por último, conviene señalar que los resultados obtenidos en este análisis no son representativos para toda la lengua, puesto que nos hemos centrado solo en una muestra de corpus, y en un género específico: el WhatsApp. En futuros estudios habría que ampliar la muestra y realizar estudios entre varios géneros, observando qué ocurre en los diferentes géneros discursivos de la lengua y en otras situaciones comunicativas. Somos conscientes de esto y este estudio solo constituye un inicio en la investigación de la estrategia lingüística de la atenuación en adolescentes y de la competencia pragmática de estos.

\section{REFERENCIAS BIBLIOGRÁFICAS}

Albelda, Marta y M $\mathrm{M}^{\mathrm{a}}$ Jesús Barros. 2013. La cortesía en la comunicación. Madrid: Arco/Libros, S. L.

Albelda, Marta, Antonio Briz, Ana María Cestero, Dorota Kotwica y Cristina Villalba. 2014. «Ficha metodológica para el análisis pragmático de la atenuación en corpus discursivos del español. (ES.POR.ATENUACIÓN)». Oralia, 17: 7-62.

Bachman, Lyle. 1990. Fundamental Considerations in Language Testing. Oxford: Oxford University Press.

Bernal, María. 2007. Categorización sociopragmática de la cortesía y de la descortesía (tesis doctoral). Estocolmo: Universidad de Estocolmo.

Bialystok, Ellen. 1993. «Symbolic Representation and Attentional Control in Pragmatic Competence». En Interlanguage Pragmatics, ed. G. Kasper y S. Blum-Kulka. Nueva York: Oxford, 43-57.

Briz, Antonio. 1998. El español coloquial en la conversación. Barcelona: Ariel.

Briz, Antonio. 2004. "Cortesía verbal codificada y cortesía verbal interpretada en la conversación». En Pragmática sociocultural: estudios sobre el discurso de cortesía en español, ed. Diana Bravo y Antonio Briz. Barcelona: Ariel, 67-94.

Briz, Antonio. 2005. «Eficacia, imagen social de cortesía. Naturaleza de la estrategia atenuadora en la conversación cotidiana española». En Estudios de la (des)cortesía 
en español. Categorías y aplicaciones a corpus orales y escritos, ed. Diana Bravo. Estocolmo: Dunken, 53-91.

Briz, Antonio y Marta Albelda. 2013. «Una propuesta teórica y metodológica para el análisis de la atenuación lingüística en español y portugués. La base de un proyecto en común (ES.POR.ATENUACIÓN)». Onomazein, 28: 288-319.

Brown, Penelope y Stephen Levinson. 1987. Politeness. Some Universals in Language Usage. Cambridge: Cambridge University Press.

Caffi, Claudia. 1999. «On mitigation». Journal of Pragmatics, 31: 881-909.

Canale, Michael y Merrill Swain. 1980. «Theoretical basis of communicative approaches to second language teaching and testing». Applied Linguistics, 1: 1-47.

Celce-Murcia, Marianne, Zoltan Dörnyei y Sarah Thurrell. 1995. «Communicative competence: A Pedagogically motivated framework with content specifications». Issues in Applied Linguistics, 6: 5-35.

Fraser, Bruce. 1980. «Conversational mitigation». Journal of Pragmatics, 4: 341-350.

González, Virginia y Amparo García. 2017. «Atenuación e intensificación: estrategias pragmáticas del rechazo en respuestas a invitaciones en redes sociales en línea». En Atenuación e intensificación en géneros discursivos, ed. M. Albelda y W. Mihatsch. Madrid: Iberoamericana Vervuert, 187-203.

Haverkate, Henk. 1994. La cortesía verbal. Estudio pragmalingüístico. Madrid: Gredos. Hymes, Dell. 1972. «On communicative competence». En Sociolinguistics, ed. J. B. Pride y J. Holmes. Harmondsworth: Penguin, 269-285.

Lakoff, Robin. 1973. "The logic of politeness; or, minding your p's and q's». Papers from the Regional Meeting. Chicago Linguistic Society, 11: 292-305.

Locher, Miriam y Richard J. Watts. 2005. «Politeness theory and relational work». Journal of Politeness Research, 1: 9-33.

Meyer-Hermann, Reinhard. 1988. «Atenuación e intensificación (análisis pragmático de sus formas y sus funciones en español hablado)». Anuario de Estudios Filológicos, 11: 275-290.

Sampietro, Agnese. 2016. «Emoticonos y multimodalidad. El uso del pulgar hacia arriba en WhatsApp». Aposta. Revista de Ciencias Sociales, 69: 271-295.

Secchi, Daniel. 2017. «La enseñanza de la atenuación en E/LE a partir del análisis de un corpus real». Foro de Profesores de E/LE, 13: 247-257. 